\title{
Pentagonal nanowires: A first-principles study of the atomic and electronic structure
}

\author{
Prasenjit Sen, ${ }^{1}$ O. Gülseren, ${ }^{2,3}$ T. Yildirim, ${ }^{2}$ Inder P. Batra, ${ }^{1}$ and S. Ciraci ${ }^{4}$ \\ ${ }^{1}$ Department of Physics, University of Illinois at Chicago, Chicago, Illinois 60607-7059 \\ ${ }^{2}$ NIST Center for Neutron Research, National Institute of Standards and Technology, Gaithersburg, Maryland 20899-8562 \\ ${ }^{3}$ Department of Materials Science and Engineering, University of Pennsylvania, Philadelphia, Pennsylvania 19104 \\ ${ }^{4}$ Department of Physics, Bilkent University, Ankara 06533, Turkey
}

(Received 27 March 2002; published 20 June 2002)

\begin{abstract}
We performed an extensive first-principles study of nanowires in various pentagonal structures by using pseudopotential plane wave method within the density functional theory. Our results show that nanowires of different types of elements, such as alkali, simple, transition, and noble metals and inert gas atoms, have a stable structure made from staggered pentagons with a linear chain perpendicular to the planes of the pentagons and passing through their centers. This structure exhibits bond angles close to those in the icosahedral structure. However, silicon is found to be energetically more favorable in the eclipsed pentagonal structure. These quasi-one-dimensional pentagonal nanowires have higher cohesive energies than many other one-dimensional structures and hence may be realized experimentally. The effects of magnetic state are examined by spinpolarized calculations. The origin of the stability is discussed by examining optimized structural parameters, charge density and electronic band structure, and by using analysis based on the empirical Lennard-Jones-type interaction. Electronic band structure of pentagonal wires of different elements are discussed and their effects on quantum ballistic conductance are mentioned. It is found that the pentagonal wire of silicon exhibits metallic band structure.
\end{abstract}

DOI: 10.1103/PhysRevB.65.235433

PACS number(s): 68.65. $-\mathrm{k}, 73.63 .-\mathrm{b}, 61.46 .+\mathrm{w}, 73.90 .+\mathrm{f}$

\section{INTRODUCTION}

Very thin metal wires produced by the tip retracting from nanoindentation in scanning tunneling microscopy (STM) or by mechanically controllable break junction (MCBJ) have been subject of a number of experimental and theoretical studies. ${ }^{1-7}$ In particular, the stepwise behavior of the conductance measured in the course of wire stretching at room temperature has attracted the interests in various fundamental features of quantum theory, such as the quantization of ballistic electron transport in very thin and one-dimensional conductors as well as Anderson's localization in very long metal wires. ${ }^{8}$ Recorded values of conductance just before the breaking of the wire were in the range of the quantum of conductance $G_{0}=2 e^{2} / h$. This implies that the smallest cross sections of the wire are of atomic dimensions. In fact, the conductance of suspended single atom gold wires, which have been produced recently, is measured to be very close to $G_{0} \cdot{ }^{9,10}$ As pointed out earlier, ${ }^{11-13}$ force and conductance variations measured concomittantly during stretching have indicated a close connection between the atomic structure and the stepwise behavior of conductance. It is now understood that a complex interplay between the quantization of electronic states with level spacing larger than room temperature, and the stable structure having well defined number of atoms ${ }^{14}$ and also dynamic self-consistent potential in presence of a current flow results in the observed step-wise behavior of conductance $G$ as a function of stretching.

Apart from being a potential nanodevice with multiple operation modes or ideal conducting connects between nanodevices, nanowires are important because of their exotic and stable atomic structures occurring in different sizes of cross sections. Therefore, a lot of effort is being devoted to the production of nanowires that are conducting and stable.
Metals crystallize in bulk three dimensional structures because that is the most stable form. If we wish to create onedimensional (1D) systems, there is clearly some struggle against nature. Furthermore, 1D periodic metals can suffer Peierls distortion and become nonmetallic. For finite nanowires, this tendency may be suppressed. However, for longer nanowires we could end up with 1D systems that are either unstable or insulating, both undesirable. Therefore, we should consider structural arrangements, 1D or perhaps quasi-1D, which have cohesive energies as close to the bulk as possible. Our search has led us to pentagonal nanowires, a quasi-1D system, where a pair of pentagons sandwich a single atom in a local icosahedral structure. The structure looks similar to a pedestal lamp with a pentagonal base, is infinitely repeated along the direction perpendicular to the planes of the pentagons, is stable, and does not suffer from Peierls distortion. We performed extensive calculations and found that these pentagonal quasi-1D nanowires have higher cohesive energies than many other 1D structural arrangements.

The pentagonal structure is incompatible with translational symmetry, and hence it is not normally seen in 2D and 3D crystal structures. Strong evidence for fivefold symmetric structures appeared in the first-principles molecular dynamics simulations where the observations of a 13 atom stable icosahedron of $\mathrm{Na}$ was reported. ${ }^{15}$ The structure can be viewed as a "tiny pentagonal nanowire" consisting of two pentagonal bases with one $\mathrm{Na}$ atom present on either side of the pentagons. The two pentagons share an apex $\mathrm{Na}$ atom and hence the $\mathrm{Na}_{13}$ cluster. Several composite structures with pentagonal motifs have also been observed in simulated annealing study of ultrathin $\mathrm{Al}$ and $\mathrm{Pb}$ nanowires. ${ }^{16}$ Subsequently, suspended monatomic chains, strands, and helical structures have been realized experimentally. ${ }^{9,10,17}$ As the fol- 
lowing discussion shows, part of the reason for the stability of the periodic pentagonal structures is that among several small planar clusters, made of particles interacting through a two-body Lennard-Jones potential, the pentagonal structure has the highest binding energy per particle.

Different regular atomic structures occurring in different sizes are now a focus of interest of experimental and theoretical studies seeking more fundamental understanding of all these structures. ${ }^{18-26}$ Whether the pentagonal structures predicted earlier by empirical methods ${ }^{16,27-29}$ are common to other elements and can be understood from more fundamental principles have become an important issue. In this paper we address this question by using the first-principles plane wave calculations within the density functional theory. We carry out state-of-the-art total energy calculations for $\mathrm{Na}, \mathrm{Al}$, $\mathrm{Cu}, \mathrm{Au}, \mathrm{Fe}, \mathrm{Ni}, \mathrm{Pb}, \mathrm{Si}$, and $\mathrm{Xe}$ in two different pentagonal structures (four structures for $\mathrm{Au}$ ), and find that the staggered pentagonal structure is a stable structure for these elements except for Si. Furthermore, we compare the energetics with other linear structures and perform an extensive analysis of the electronic structure and charge density to reveal the origin of stability and electronic properties of the pentagonal structure. Finally, we mention the effect of the pentagonal structure on the ballistic conductance.

\section{DESCRIPTION OF THE METHOD AND ATOMIC STRUCTURE}

First-principles plane wave calculations are performed within the supercell geometry using a tetragonal unit cell. The axis of the wire is taken along the $z$ axis, and the lattice parameter of the wire coincides with the lattice parameter $c$ of the tetragonal supercell. The lattice parameters of the tetragonal cell in the $x-y$ plane are set as $a=b=15 \AA$ so that the interaction between a wire and its periodic images are negligible. Bloch states are expressed by the linear combinations of plane waves with the cutoff energy $|\mathbf{k}+\mathbf{G}|^{2}$ always larger than the optimum cutoff energy suggested for the ionic ultrasoft pseudopotential ${ }^{30}$ of the element under study. The Brillouin zone (BZ) integration is performed within Monkhorst-Pack scheme ${ }^{31}$ using $(1 \times 1 \times 20) \mathbf{k}$ points. Results are obtained by generalized gradient approximation ${ }^{32}$ (GGA). Preconditioned conjugate gradient (CG) method is used for wave function optimization. To find the correct ground state we also performed spin-polarized calculations for nanowires of $\mathrm{Fe}$ and Ni. Numerical calculations are performed using both VASP (Ref. 33) and CASTEP (Ref. 34) codes independently.

We considered the following pentagonal structures. (i) Atoms form parallel pentagons which are perpendicular to the $(z)$ axis of the wire with separation $w$, but successive pentagons are rotated by $\pi / 5$. In addition, a monatomic chain along the $z$ axis passes through the center of pentagons, where each chain atom is located at a point equidistant from the planes of pentagons. The lattice parameter $c$ in the direction of the chain is twice the spacing between pentagons, i.e., $c=2 w$. This structure is specified as the staggered pentagon $\mathcal{S}$ and has 12 atoms in the unit cell. (ii) Same as (i), but successive pentagons are not rotated so that they have the
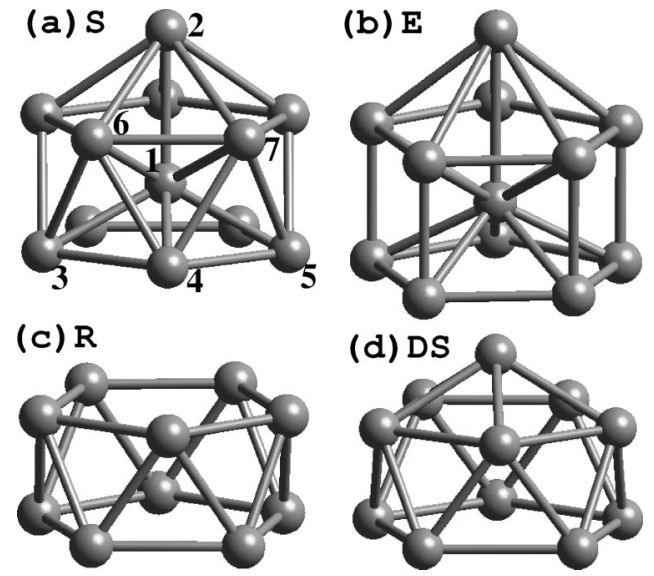

FIG. 1. Schematic description of various pentagonal structures with the structural parameters: Lattice parameter along the wire $c$ and spacing between adjacent pentagons $w$. (a) $\mathcal{S}$ staggered pentagon structure with $c=2 w$. Numerals specify atoms. Atoms 1 and 2 form the chain passing through the centers of the staggered pentagons. Relevant interatomic distances $d_{C-C}=w, d_{C-P}, d_{P-P}$, and $d_{P_{1}-P_{2}}$ are between the atoms (1-2), (1-3), (3-4), and (4-6), respectively. Bond angles $\alpha_{1}, \alpha_{2}, \alpha_{3}, \alpha_{4}$, and $\alpha_{5}$ occur between (3-1-4), (3-1-5), (3-1-6), (3-1-7), and (3-6-4). (b) In the eclipsed pentagon structure $\mathcal{E}$ all the pentagons are aligned. Hence $d_{C-C}=d_{P_{1}-P_{2}}=w$ and $c=w$. (c) Staggered pentagonal structure $\mathcal{R}$ is similar to $\mathcal{S}$ structure in (a), except that central atomic chain is missing $c=2 w$. (d) The deformed staggered pentagon structure $\mathcal{D S}$ with $c=2 w$.

same orientation relative to the $z$ axis, and hence $c=w$. This structure is the eclipsed pentagon, $\mathcal{E}$ with six atoms per unit cell. (iii) Staggered pentagonal structure without the monatomic chain passing through the centers of the parallel pentagons. This is called the $\mathcal{R}$ structure and has $c=2 w$. (iv) We also found a modified version of the $\mathcal{S}$ structure in gold nanowires, which is specified as the deformed staggered pentagon, $\mathcal{D S}$. Here, adjacent pentagons are staggered, but one of the two atoms of the central chain in a unit cell is slightly displaced, while the other one is missing. Accordingly, $\mathcal{R}$ and $\mathcal{D S}$ structures have 10 and 11 atoms in a unit cell, respectively. The pentagonal structures and their relevant structural parameters are schematically described in Fig. 1.

\section{RESULTS AND DISCUSSIONS}

\section{A. Optimized structures and energetics}

The energetics and atomic structure of $\mathrm{Na}, \mathrm{Al}, \mathrm{Pb}, \mathrm{Cu}, \mathrm{Au}$, $\mathrm{Ni}, \mathrm{Fe}, \mathrm{Si}$, and Xe wires in $\mathcal{S}$ and $\mathcal{E}$ have been investigated. Our results for the structural parameters and binding energies are listed in Table I. The binding energies and the relevant interatomic distances corresponding to equilibrium bulk crystal structures are also given for the sake of comparison. The binding energy per atom for a given structure is calculated as the difference of the energy $E_{a}$ of an individual atom and the total energy of wire $E_{T}$ having $n$ atoms in the supercell divided by $n$, i.e., $E_{B}=E_{a}-E_{T} / n$. In spite of the fact that $\mathrm{Na}, \mathrm{Al}, \mathrm{Cu}, \mathrm{Pb}, \mathrm{Au}, \mathrm{Fe}, \mathrm{Ni}, \mathrm{Si}$, and $\mathrm{Xe}$ atoms have different electronic configurations and form bulk crystals with dramatically different properties, they all form stable 
TABLE I. Comparison of calculated structural parameters and binding energy $E_{B}$ for different pentagonal structures of different elements. The nearest neighbor distance $d_{0}$ and binding energy $E_{0}$ are calculated for the optimized bulk crystals. $S, E, R$, DS, and SM are staggered, eclipsed, staggered without central chain, deformed staggered, and magnetic staggered, respectively. Bond lengths and energies are in $\AA$ and $\mathrm{eV}$, respectively.

\begin{tabular}{|c|c|c|c|c|c|c|c|c|c|c|c|c|c|}
\hline Atom & Structure & $d_{C-C}$ & $d_{C-P}$ & $d_{P-P}$ & $d_{P_{1}-P_{2}}$ & $\alpha_{1}$ & $\alpha_{2}$ & $\alpha_{3}$ & $\alpha_{4}$ & $\alpha_{5}$ & $E_{B}(\mathrm{eV})$ & $d_{0}$ & $E_{0}$ \\
\hline $\mathrm{Na}$ & $S$ & 3.02 & 3.75 & 4.1 & 3.67 & 65 & 114.5 & 59 & 114.5 & 66 & 1.054 & 3.53 & 1.28 \\
\hline $\mathrm{Na}$ & $E$ & 3.46 & 3.77 & 3.95 & 3.46 & 63 & 115 & 55.3 & 87 & 47.3 & 0.989 & & \\
\hline $\mathrm{Al}$ & $S$ & 2.39 & 2.71 & 2.86 & 2.82 & 63.7 & 117.2 & 62.7 & 116.2 & 61 & 3.201 & 2.80 & 3.766 \\
\hline $\mathrm{Al}$ & $E$ & 2.54 & 2.76 & 2.88 & 2.54 & 63.1 & 115.3 & 54.8 & 88.5 & 48.6 & 3.189 & & \\
\hline $\mathrm{Al}$ & $R$ & & & 2.68 & 2.63 & & & & & 61.3 & 3.057 & & \\
\hline $\mathrm{Al}$ & DS & 4.54 & 2.62 & 2.77 & 2.70 & 63.9 & 118 & 62 & 116 & 58 & 3.21 & & \\
\hline $\mathrm{Cu}$ & $S$ & 2.21 & 2.48 & 2.61 & 2.61 & 63.4 & 116.9 & 63.5 & 116.7 & 60.2 & 3.017 & 2.58 & 3.76 \\
\hline $\mathrm{Cu}$ & $E$ & 2.46 & 2.48 & 2.53 & 2.46 & 61.5 & 111.3 & 59.5 & 91 & 46 & 2.878 & & \\
\hline $\mathrm{Pb}$ & $S$ & 3.41 & 3.29 & 3.38 & 3.45 & 61 & 111 & 63 & 114 & 58 & 3.18 & 3.56 & 3.51 \\
\hline $\mathrm{Pb}$ & $E$ & 3.56 & 3.32 & 3.28 & 3.56 & 59.5 & 106.5 & 65.1 & 93.5 & 43 & 3.13 & & \\
\hline $\mathrm{Au}$ & $S$ & 2.50 & 2.88 & 3.05 & 2.97 & 63.8 & 117.8 & 62.4 & 115.9 & 61.7 & 2.526 & 2.95 & 3.211 \\
\hline $\mathrm{Au}$ & $E$ & 2.76 & 2.87 & 2.98 & 2.76 & 61.8 & 113.7 & 57.1 & 89 & 47.8 & 2.494 & & \\
\hline $\mathrm{Au}$ & $R$ & & & 2.89 & 2.74 & & & & & 63.4 & 2.662 & & \\
\hline \multirow[t]{2}{*}{$\mathrm{Au}$} & DS & 4.51 & 2.81 & 3.15 & 2.80 & 68 & 127.5 & 58.2 & 113.5 & 69 & 2.669 & & \\
\hline & & & 2.88 & 2.98 & 2.75 & 62.5 & 113.5 & & & & & & \\
\hline $\mathrm{Fe}$ & SM & 2.20 & 2.50 & 2.62 & 2.61 & 63.5 & 117.2 & 63 & 117 & 61 & 7.298 & 2.48 & 8.37 \\
\hline $\mathrm{Fe}$ & $S$ & 2.16 & 2.31 & 2.39 & 2.51 & 62.4 & 114.2 & 65.8 & 117.5 & 57 & 6.563 & & \\
\hline $\mathrm{Ni}$ & $\mathrm{SM}$ & 2.19 & 2.40 & 2.51 & 2.56 & 63.1 & 115.5 & 64.4 & 117.1 & 58.7 & 4.444 & 2.49 & 5.48 \\
\hline $\mathrm{Ni}$ & $S$ & 2.16 & 2.40 & 2.52 & 2.53 & 63.4 & 116.5 & 63.8 & 117 & 59.7 & 4.337 & & \\
\hline $\mathrm{Ni}$ & $E$ & 2.33 & 2.38 & 2.43 & 2.33 & 62.5 & 112.3 & 58.6 & 89.8 & 46.1 & 4.20 & & \\
\hline $\mathrm{Si}$ & $S$ & 2.62 & 2.57 & 2.60 & 2.96 & 60.7 & 109.8 & 70.1 & 119.3 & 52.2 & 4.524 & 2.35 & 5.40 \\
\hline $\mathrm{Si}$ & $E$ & 2.73 & 2.56 & 2.55 & 2.73 & 59.6 & 107.3 & 64.2 & 93.6 & 43.1 & 4.592 & & \\
\hline $\mathrm{Xe}$ & $S$ & 3.74 & 4.05 & 4.23 & 4.36 & 62.9 & 115.1 & 65 & 117.3 & 58.1 & 0.143 & 4.51 & 0.06 \\
\hline $\mathrm{Xe}$ & $E$ & 4.04 & 4.05 & 4.85 & 4.04 & 73 & 142 & 46.8 & 81 & 50 & 0.104 & & \\
\hline
\end{tabular}

wires in the pentagonal structure. It is very interesting that $\mathrm{Al}$ with $3 s^{2} 3 p$ valence states and Na with $3 s$ valence states form similar pentagonal structures. Silicon, a group IV element which is normally crystallized in (tetrahedrally bonded) diamond structure is predicted to form pentagonal wires similar to what Xe, having a closed shell structure, does. The pentagonal structure is a stable structure corresponding to a local minimum on the Born-Oppenheimer surface.

Calculated binding energies show that among the pentagonal $\mathcal{S}$ and $\mathcal{E}$ structures the staggered one is energetically more favorable for $\mathrm{Na}, \mathrm{Al}, \mathrm{Cu}, \mathrm{Pb}, \mathrm{Au}, \mathrm{Fe}, \mathrm{Ni}$, and $\mathrm{Xe}$. However, the differences in binding energies $\Delta E_{B}=E_{B, \mathcal{S}}$ $-E_{B, \mathcal{E}}$ are generally small and are in the range of $\sim 10 \mathrm{meV} . \Delta E_{B}<0$ for $\mathrm{Si}$, which favors the eclipsed pentagonal structure.

We investigated the rotation between $\mathcal{S}$ and $\mathcal{E}$ structures of gold nanowires by breaking the rotation from $\mathcal{S}$ to $\mathcal{E}$ in seven steps; at each step the relative angle $(\varphi)$ between two pentagons in a unit cell increased by an angle $\Delta \varphi=6^{\circ}$. For 0 $\leqslant \varphi<36^{\circ}$ the lattice parameter $c$ is twice the spacing between pentagons $w$, i.e., $c=2 w$. The variation of the energy as a function of the rotation angle $\varphi$ is illustrated in Fig. 2. The maximum of the energy curve corresponds to $Q_{\mathcal{S} \rightarrow \mathcal{E}}$. For robust rotation of pentagons, the wire is apparently under

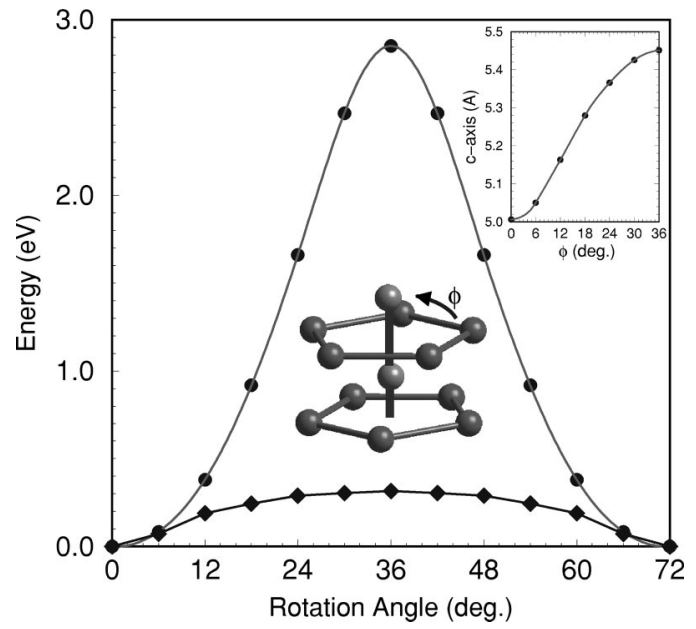

FIG. 2. Variation of the total energy with the rotation angle $\varphi$ from $\mathcal{S}$ structure $(\varphi=0)$ to $\mathcal{E}$ structure $\left(\varphi=36^{\circ}\right)$. The maximum energy is the energy difference $Q_{\mathcal{S} \rightarrow \mathcal{E}}$ between the $\mathcal{S}$ and the $\mathcal{E}$ structures. Energies indicated by dots correspond to the robust rotation of pentagons without relaxation of the structure. Energies indicated by diamonds are calculated by fully optimizing the structure including the lattice parameters at each step. The inset shows variation of $c$. 
high compression due to the core-core repulsion between two adjacent pentagons, and consequently $Q_{\mathcal{S} \rightarrow \mathcal{E}}$ acquires a high value $\sim 3 \mathrm{eV}$. However, upon optimizing the structure after each rotation step, the structure is modified, in particular, the spacing between adjacent pentagons increases with $\varphi$. As a result, the energies calculated are lowered dramatically, the curve for the variation of the energy as a function of $\varphi$ is flattened and $Q_{\mathcal{S} \rightarrow \mathcal{E}}$ is reduced to $\sim 0.38 \mathrm{eV}$ per cell (or $32 \mathrm{meV} /$ atom). We note that for $30^{\circ}<\varphi<42^{\circ}$ around the $\mathcal{E}$ structure the energy curve is practically flat. This implies that $\mathcal{E}$ structure may acquire a helicity along the axis of the wire, if each pentagon rotates by a small angle $\varphi$. In fact, the helicity in nanowires was seen in classical MD calculations ${ }^{16}$ and has recently been observed experimentally. ${ }^{17}$ Nevertheless, it is clear that the $\mathcal{E}$ structure is not only energetically less favorable, but is also unstable.

From Table I it is easily seen that this is the general trend in all systems that show metallic bonding $(\mathrm{Na}, \mathrm{Al}, \mathrm{Cu}, \mathrm{Pb}$, $\mathrm{Au}, \mathrm{Ni}$ ). Si also shows a similar behavior, but the relaxation of the $z$ axis lattice constant in rotating the nanowire from the $\mathcal{S}$ to the $\mathcal{E}$ structure is much less compared to $\mathrm{Na}, \mathrm{Cu}$, or $\mathrm{Au}$. This is because, in a system with metallic bonding, the electrons are largely delocalized and they screen the ion cores less effectively. Hence, when the cores of the atoms in two successive pentagons come closer on the structure being rotated from $\mathcal{S}$ to $\mathcal{E}$, there is strong repulsion which tends to increase $w$ and make the latter structure less favorable. For a system such as $\mathrm{Si}$, showing directional bonds, there is much better screening of the cores. In the $\mathcal{S}$ structure, each $\mathrm{Si}$ atom in the pentagon forms four bonds-two bonds of $2.57 \AA$ with two central chain atoms and two bonds of $2.60 \AA$ with two other atoms in the pentagon. The third neighbor of a $\mathrm{Si}$ atom is $2.96 \AA$ apart, and there is no bond formation with it. On the other hand, for a $\mathrm{Si}$ atom in the pentagon for the $\mathcal{E}$ structure, there are six bonds-two bonds of $2.56 \AA$ with two central chain atoms, two bonds of $2.55 \AA$ with two atoms in the pentagon and two weaker bonds of $2.73 \AA$ with two $\mathrm{Si}$ atoms in the pentagons above and below it.

In course of structure optimization of gold wire, we found two other structures- $\mathcal{R}$ and $\mathcal{D S}$ structures (see Fig. 1). These structures have smaller numbers of atoms in their unit cells, and are found to be energetically more favorable than the $\mathcal{S}$ structure in the case of gold nanowire. Interestingly, $\mathcal{R}$ is found to be energetically less favorable than $\mathcal{S}$ for $\mathrm{Al}$ nanowires. The stability of the $\mathcal{S}$ structure is examined for transition metals $\mathrm{Ni}$ and $\mathrm{Fe}$ by performing both spin unpolarized and spin polarized calculations. We found that $\mathrm{Ni}$ and Fe nanowires in staggered pentagon structures are stable for both nonmagnetic (spin unpolarized) and magnetic (spin polarized) states. Although, for Ni, the energy gain in the magnetic structure compared to the nonmagnetic one is small, the spin polarized state of $\mathrm{Fe}$ staggered pentagonal wire increases the binding energy by $0.73 \mathrm{eV} /$ atom. Similarly, the magnetic moment per atom is also much larger in case of $\mathrm{Fe}$ $\left(\sim 3 \mu_{B}\right)$ than in $\mathrm{Ni}\left(\sim 0.77 \mu_{B}\right)$.

The interatomic distance from the chain atom to the corners of a pentagon $d_{C-P}$ is slightly smaller than the nearest neighbor distance within the pentagon $d_{P-P}$ but both distances are close to the nearest neighbor distance $d_{0}$ of the
TABLE II. Comparison of nearest-neighbor distance and binding energies of monatomic linear $(L)$, zigzag $(W)$, triangular $(T)$ chain structures, and bulk crystal $(B)$ with the binding energy of the staggered pentagon $\mathcal{S}$ structure calculated for $\mathrm{Al}$ and $\mathrm{Au}$. The binding energies of $L, W$, and $T$ structures are taken from Ref. 24 .

\begin{tabular}{lcccc}
\hline \hline & \multicolumn{3}{c}{ Aluminum } & \multicolumn{2}{c}{ Gold } \\
& $d(\AA)$ & $E_{b}(\mathrm{eV} /$ atom $)$ & $d(\AA)$ & $E_{b}(\mathrm{eV} /$ atom $)$ \\
\hline$L$ & 2.41 & 1.87 & 2.59 & 1.68 \\
$W$ & 2.53 & 1.92 & 2.56 & 1.90 \\
$T$ & 2.51 & 2.65 & 2.71 & 2.23 \\
$S$ & 2.71 & 3.20 & 2.88 & 2.53 \\
$B$ & 2.80 & 3.76 & 2.95 & 3.21 \\
\hline \hline
\end{tabular}

bulk crystal. Assuming that $d_{C-P} \approx d_{P-P}$, the coordination number for a chain atom is equal to 10 , and that for an atom of the pentagon is 4 . Hence, roughly speaking, the average coordination number is 7 . This is smaller than the bulk fcc and bcc coordination numbers 12 and 8 , respectively. We note, however, the nearest neighbor distance between adjacent pentagons $d_{P_{1}-P_{2}} \sim 14-20 \%$ larger than $d_{C-P}$ and $d_{P-P}$. The bond angles $\alpha_{1}-\alpha_{5}$, are close to the bond angles of icosahedral structure, i.e., $63.4^{\circ}$ and $116.6^{\circ}$. Therefore, the local atomic configuration in the pentagonal wires mimics the icosahedral structure. ${ }^{16,35}$

Calculated binding energies $E_{B}$ of pentagonal wires are lower than the calculated bulk binding energies $E_{0}$ in the last column of Table I. This can be explained by higher coordination number in bulk crystals. On the other hand, the binding energies of pentagonal structure are higher than the binding energies of various monatomic chain structures. In Table II the binding energies of $\mathcal{S}$ structure are compared with the binding energies of relevant monatomic chain structures calculated $^{24}$ earlier for $\mathrm{Al}$ and $\mathrm{Au}$. For example, three monatomic chain structures of $\mathrm{Al}$ are linear $(L)$, zigzag $(W)$, and equilateral triangular $(T)$ chains have binding energies 1.87 , 1.92 , and $2.50 \mathrm{eV}$, respectively. The coordination number of the $\mathcal{S}$ structure has an intermediate value between those of 1D monatomic chain structures and bulk crystal, so its binding energy $E_{B}=3.2 \mathrm{eV}$. Gold also follows the same ordering. We note the general trend that the binding energy increases with increasing coordination number in different structures. This trend is clearly observed in Table II by going from the monatomic linear chain to the bulk. However, in the Xe pentagonal wires, the binding energy becomes larger than the binding energy of the bulk fcc structure (excluding the contribution of the van der Waals interaction). On the other hand, all the nearest neighbor distances $\left(d_{C-C}, d_{C-P}, d_{P-P}\right.$, etc.) are much smaller than the bulk nearest neighbor distance $d_{0}$.

\section{B. Energy band structure and total charge density}

The electronic energy band structure of $\mathrm{Na}, \mathrm{Al}, \mathrm{Au}$, and $\mathrm{Si}$ are presented for both $\mathcal{S}$ and $\mathcal{E}$ structures in Fig. 3. Overall forms of the energy bands are the same in both structures for $\mathrm{Na}, \mathrm{Al}$, and $\mathrm{Au}$, except for some shifts and splittings of de- 

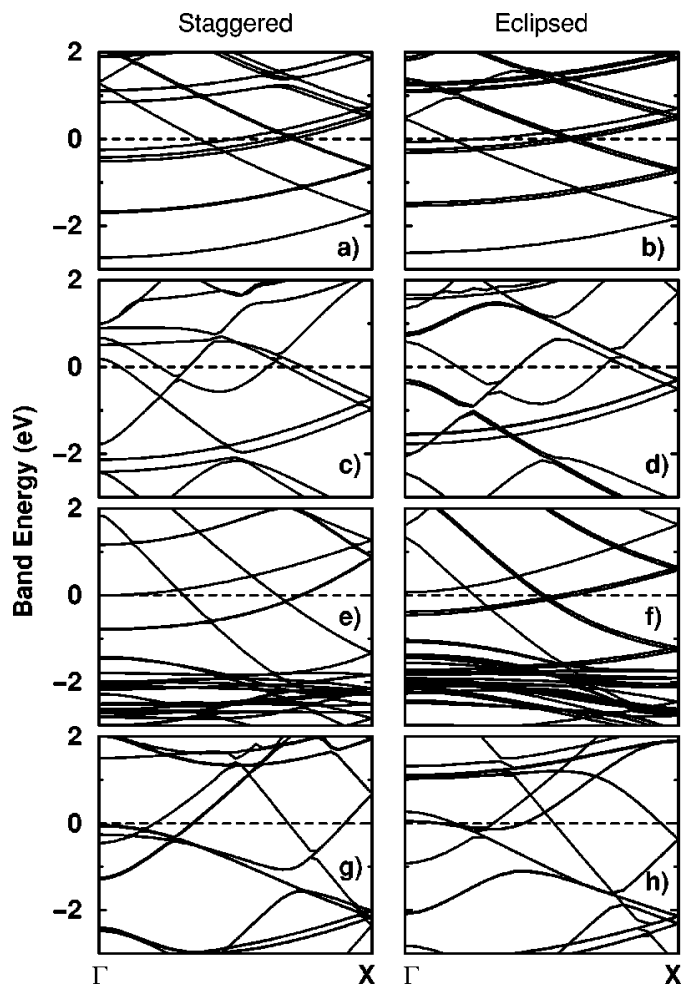

FIG. 3. Energy band structures of $\mathcal{S}$ and $\mathcal{E}$ structures, respectively, (a) and (b) for $\mathrm{Na}$, (c) and (d) for $\mathrm{Al}$, (e) and (f) for $\mathrm{Au}$, and (g) and (h) for Si. Fermi level shown by dashed lines marks the zero of energy.

generate bands. All these structures have the same number of atoms in their cells, but Fermi level is crossed by different number of energy bands for different elements. Number of bands which cross the Fermi level is crucial for the quantum ballistic conductance and the stability of nanowire. Under ideal conditions, the conductance is determined by the number of bands crossing the Fermi level, being $G_{0}$ per band. We found that the stable staggered pentagon structures of $\mathrm{Na}, \mathrm{Al}$, and $\mathrm{Au}$ nanowires have six, ten and six bands crossing the Fermi level, respectively. On the other hand, the Fermi level of the unstable eclipsed pentagon structure of $\mathrm{Na}, \mathrm{Al}$, and $\mathrm{Au}$ nanowires is crossed by six, eight, and five bands, respectively. While the staggered pentagonal structure of Si nanowire have six bands crossing the Fermi level, this number is raised to ten for the eclipsed pentagon, which is the stable structure. One notices that a degenerate band has dropped below the Fermi energy in going from the $\mathcal{S}$ to the $\mathcal{E}$ structure for Si. This is possibly because of a weak bond formation between $\mathrm{Si}$ atoms on different pentagonal planes, which is absent in the $\mathcal{S}$ structure, and is responsible for the $\mathcal{E}$ structure being more stable. While stretching the nanowire, the number of atoms in the neck region, where the wire is thinned, and their structure exhibit sequential and stepwise changes. ${ }^{13,27}$ It has been argued that these changes are closely related with a band moving up from the Fermi level and becoming unoccupied. ${ }^{14,36}$ An important feature of Fig. 3 is that the Si nanowire is metallic in both structures. Several bands crossing the Fermi level gives rise to high density of states at $E_{F}$. We found that all pentagonal nanowires except
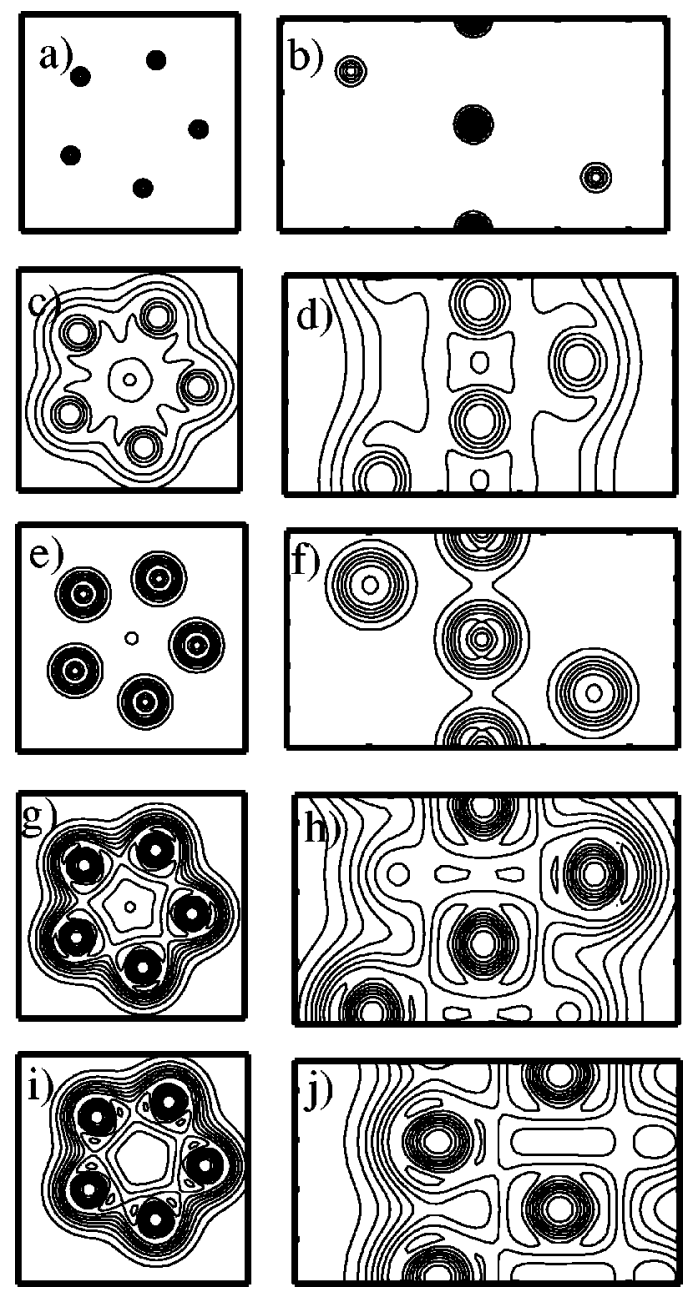

FIG. 4. Left panels are charge density contour plots in lateral planes which coincide with the plane of pentagons. Right panels are in vertical planes which pass through the central chain and one atom of each pentagon. (a) and (b): $\mathrm{Na}$ in $\mathcal{S}$ structure. (c) and (d): Al in $\mathcal{S}$ structure. (e) and (f): Au in $\mathcal{S}$ structure. (g) and (h) $\mathrm{Si}$ in $\mathcal{S}$ structure. (i) and (j): $\mathrm{Si}$ in $\mathcal{E}$ structure.

Xe studied in this paper are metallic. The Xe nanowire in $\mathcal{S}$ structure is a semiconductor with a wide band gap.

The character of the bonding in pentagonal structures is revealed by the analysis of electronic charge density. In Fig. 4 we show the charge density contour plots of $\mathrm{Na}, \mathrm{Al}, \mathrm{Au}$, and $\mathrm{Si}$ in different planes. The lateral plane passing through the plane of the pentagon shows the character of the bonding between the atoms in a pentagon. The vertical plane includes the central chain as well as one atom of the pentagon. Because of inversion symmetry of the $\mathcal{S}$ structure, one atom of the adjacent pentagon is also included in the same vertical plane. The charge distribution of $\mathrm{Na}$ is uniform in vertical and lateral planes. Because of low charge density significant structure cannot be resolved. The charge density contour plots in Fig. 4 display some differences in the charge distributions between different atoms. The charge distribution of the central chain for $\mathrm{Al}$ is reminiscent of that of monatomic chain structure ${ }^{24}$ and has a directional character. The directional behavior is, however, less pronounced in the bonds 
forming between two atoms in the same pentagon and also between central chain atom and pentagon atom. The charge distribution of gold wire reflects the charge distribution of bulk metal. The directionality with high density along the line connecting two nearest neighbor atoms is absent. Nonuniform charge distribution with a directionality between nearest neighbor atoms is clearly seen in the contour plots of Si both in $\mathcal{S}$ and $\mathcal{E}$ structures. The directionality of charge distribution originates from the valence of the element which makes the pentagonal wire. Here, $\mathrm{Al}$ and $\mathrm{Si}$ with valence states consisting of $3 s, 3 p_{x, y, z}$ orbitals form directional bonds. In contrast, $\mathrm{Na}$ and $\mathrm{Au}$ with $s$ valence orbitals exhibit bulklike, uniform charge distribution which is characteristic of metals. Despite these differences, all the pentagonal nanowires in Fig. 4 are metals with finite density of states at the Fermi level.

\section{Discussions}

As corroborated by the present first-principles calculations, the pentagonal structure is one of the energetically favorable structures of wires having translational periodicity along its axis. Of course, it is only a local minimum on the Born-Oppenheimer surface and occurs for a given number of atoms in the cross section of the wire (or in the 1D unit cell). Since the pentagonal structure occurs for a number of different elements as demonstrated in this study, the stability must stem from the pentagonal geometry. We examined the relative stability of the pentagonal geometry by performing a simple analysis based on the two-body Lennard-Jones potential $V\left(r_{i, j}\right)=4 \epsilon\left[\left(\sigma / r_{i, j}\right)^{12}-\left(\sigma / r_{i, j}\right)^{6}\right]$. We considered three structures encountered in the structure optimization of very thin wires, ${ }^{16,27}$ namely, equilateral triangle, pentagon, and hexagon. The total potential $V_{T}=\Sigma_{i \neq j} V_{i, j}\left(r_{i, j}\right)$ for these structures are obtained by adding all the two-body potential energies $V_{i, j}$. By optimizing the total potential energy $V_{T}$ relative to the nearest neighbor distance $d_{1}, \partial V_{T} / \partial d_{1}=0$, the optimized interatomic distances and the binding energies for these three structures are calculated in terms of the parameters $\sigma$ and $\epsilon$. We found binding energies $E_{b}$ of $-\epsilon$, $-1.1111 \epsilon$, and $-1.09 \epsilon$, for equilateral triangle, pentagon and hexagons, respectively. The corresponding optimized nearest neighbor distances are $2^{1 / 6} \sigma, 1.89 \sigma$, and $1.91 \sigma$, respectively. It is very interesting to note that among these structures, the pentagon has the highest binding energy.

\section{CONCLUSIONS}

We carried out an extensive analysis of the energetics and structure of thin wires made from different elements, such as alkali, simple, noble, and transition metals, and also Si, Xe. First-principles calculations with fully optimized structures yield that the 1D structure formed by parallel but staggered pentagons and an atomic chain passing through the center of pentagons is generally stable and energetically favorable relative to other pentagonal structures. However, there are exceptions. For example, while the eclipsed pentagonal structure is favored by Si nanowires, in gold wires, different versions of pentagonal structures are found to be energetically more favorable. The binding energies are intermediate between 1D chain structure and bulk crystal. All nanowires of different elements studied in this paper, except Xe, are metallic in the pentagonal structure. Strong cohesion and metallicity of quasi-1D pentagonal nanowires suggest that they can be useful in practical applications and deserve further experimental studies.

\section{ACKNOWLEDGMENTS}

This work was partially supported by the NSF under Grant No. INT01-15021 and TÜBÍTAK under Grant No. TBAG-U/13(101T010).
${ }^{1}$ S. Ciraci, A. Baratoff, and Inder P. Bartra, Phys. Rev. B 41, 2763 (1990).

${ }^{2}$ N. Agraï, J.G. Rodrigo, and S. Vieira, Phys. Rev. B 47, 12345 (1993).

${ }^{3}$ J.M. Krans, C.J. Müller, I.K. Yanson, T.C.M. Gowarent, R. Hesper, and J.M. van Ruitenbeek, Phys. Rev. B 50, 17659 (1994).

${ }^{4}$ J.I. Pascual, J. Mendez, J. Gomez-Herrero, A.M. Baro, N. Garcia, and V.T. Binh, Phys. Rev. Lett. 71, 1852 (1993).

${ }^{5}$ O. Tomagnini, F. Ercolessi, and E. Tosatti, Surf. Sci. 287-288, 1041 (1993).

${ }^{6}$ L. Kuipers and J.W.M. Frenken, Phys. Rev. Lett. 70, 3907 (1993).

${ }^{7}$ A current review of the subject can be found in S. Ciraci, A. Buldum, and I.P. Batra, J. Phys.: Condens. Matter 13, R537 (2001).

${ }^{8}$ J.I. Pascual, J. Mendez, J. Herrero-Gomez, A.M. Baro, N. Garcia, U. Landman, W.D. Luedtke, E.N. Bogachek, and H.P. Cheng, Science 267, 1793 (1995).

${ }^{9}$ H. Ohnishi, Y. Kondo, and K. Takayanagi, Nature (London) 395, 780 (1998).
${ }^{10}$ A.I. Yanson, G.R. Bolliger, H.E. van der Brom, N. Agraï, and J.M. van Ruitenbeek, Nature (London) 395, 783 (1998).

${ }^{11}$ S. Ciraci and E. Tekman, Phys. Rev. B 40, R11 969 (1989); E. Tekman and S. Ciraci, ibid. 43, 7145 (1991).

${ }^{12}$ T.N. Todorov and A.P. Sutton, Phys. Rev. Lett. 70, 2138 (1993).

${ }^{13}$ N. Agrait, G. Rubio, and S. Vieira, Phys. Rev. Lett. 74, 3995 (1995); G. Rubio, N. Agrait, and S. Vieira, ibid. 76, 2302 (1996).

${ }^{14}$ C.A. Stafford, D. Baeriswyl, and J. Bürki, Phys. Rev. Lett. 79, 2863 (1997).

${ }^{15}$ R.N. Barnett and U. Landman, Nature (London) 387, 788 (1997).

${ }^{16}$ O. Gülseren, F. Ercolessi, and E. Tosatti, Phys. Rev. Lett. 80, 3775 (1998).

${ }^{17}$ Y. Kondo and K. Takayanagi, Science 289, 600 (2000).

${ }^{18}$ J.A. Torres, E. Tosatti, A. Dal Corso, F. Ercolessi, J.J. Kohanoff, F.D. Di Tolla, and J.M. Soler, Surf. Sci. 426, L441 (1999).

${ }^{19}$ L. De Maria and M. Springborg, Chem. Phys. Lett. 323, 293 (2000).

${ }^{20}$ H. Häkkinen, R.N. Barnett, A.G. Scherbakov, and U. Landman, J. Phys. Chem. B 104, 9063 (2000). 
${ }^{21}$ F.D. Tolla, A.D. Corsa, J.A. Torres, and E. Tosatti, Surf. Sci. 454-456, 947 (2000).

${ }^{22}$ M. Okamoto and K. Takayanagi, Phys. Rev. B 60, 7808 (1999).

${ }^{23}$ D. Sanchez-Portal, E. Artacho, J. Junquera, P. Ordejon, A. Garcia, and J.M. Soler, Phys. Rev. Lett. 83, 3884 (1999).

${ }^{24}$ P. Sen, S. Ciraci, A. Buldum, and I.P. Batra, Phys. Rev. B 64, 195420 (2001).

${ }^{25}$ I. P. Batra, P. Sen, and S. Ciraci, J. Vac. Sci. Technol. B 20, 812 (2002)

${ }^{26}$ B.L. Wang, S.Y. Yin, G.H. Wang, A. Buldum, and J.J. Zhao, Phys. Rev. Lett. 86, 2046 (2001); B.L. Wang, S.Y. Yin, G.H. Wang, and J.J. Zhao, J. Phys.: Condens. Matter 13, 403 (2001).

${ }^{27}$ H. Mehrez and S. Ciraci, Phys. Rev. B 56, 12632 (1997); H. Mehrez, S. Ciraci, A. Buldum, and I.P. Batra, ibid. 55, R1981 (1998).

${ }^{28}$ M. Brandbyge, K.W. Jacobsen, and J.K. Norskov, Phys. Rev. B
55, 2637 (1997); M. Brandbyge, M.R. Sorensen, and K.W. Jacobsen, ibid. 56, 14956 (1997).

${ }^{29}$ J.W. Kang and H.J. Hwang, J. Phys.: Condens. Matter 14, 2629 (2002).

${ }^{30}$ D. Vanderbilt, Phys. Rev. B 41, 7892 (1990).

${ }^{31}$ H.J. Monkhorst, and J.D. Pack, Phys. Rev. B 13, 5188 (1976).

${ }^{32}$ J.P. Perdew et al., Phys. Rev. B 46, 6671 (1992).

${ }^{33}$ G. Kresse and J. Hafner, Phys. Rev. B 47, R558 (1993); G. Kresse and J. Furthmüller, ibid. 54, 11169 (1996).

${ }^{34}$ M.C. Payne, M.P. Teter, D.C. Allen, T.A. Arias, and J.D. Joannopoulos, Rev. Mod. Phys. 64, 1045 (1992).

${ }^{35}$ S. Ino, J. Phys. Soc. Jpn. 27, 941 (1969).

${ }^{36}$ S. Ciraci and I.P. Batra, Phys. Rev. B 33, 4294 (1986); I.P. Batra, S. Ciraci, G.P. Srivastava, J.S. Nelson, and C.Y. Fong, ibid. 34, 8246 (1986). 